\title{
Perioperative Maßnahmen am Beispiel des alten Menschen
}

\author{
Synke Petschinka, Regina Ulrike Dries, Oliver Hauschild, Norbert Paul Südkamp
}

\section{Zusammenfassung}

Die perioperative Pflege des alten $\mathrm{Pa}$ tienten beginnt schon mit der Entscheidung zur Durchführung einer Operation. Unter perioperativer Pflege versteht man die Vernetzung und $\mathrm{Zu}-$ sammenfassung von Pflegetätigkeiten in den Phasen vor, während und nach einer Operation. Die perioperative Pflege beinhaltet die Vorbereitung und Betreuung der Patienten, die zur Operation anstehen, alle pflegerischen Tätigkeiten während des operativen Eingriffs und die durchzuführenden Tätigkeiten bis zur Übergabe des Patienten an die Station. Hier ist ein strukturierter Pflegeprozess von Vorteil, damit Ablauf und Struktur im OP für alle Mitarbeiter nachvollziehbar und transparent sind. Ein offener Informationsaustausch zwischen Chirurg, Patient, OP-Pflege und Anästhesie muss gewährleistet sein. Für den alten Patienten ist dies von besonderer Bedeutung, da das Ergebnis der Operation zu einschneidenden Veränderungen im Alltag führen kann. Alle möglichen positiven wie negativen Operationskonsequenzen müssen vorher ins Auge gefasst und besprochen werden. Um die bestmögliche Behandlung zu gewährleisten, ist es erforderlich, in den Vorbereitungsphasen optimale Arbeitsbedingungen sowohl für das chirurgische als auch für das anästhesiologische Team und deren Interaktion zu schaffen. Erfahrungsgemäß löst ein anstehender operativer Eingriff bei älteren Patienten häufig ein Gefühl der Unsicherheit und Angst aus, ein Umstand, dem im perioperativen Pflegeprozess Rechnung getragen werden sollte. Leider reicht oft die kurze verbleibende Zeit vor der OP nicht aus, um hinreichend empathisch und umsorgend auf den Patienten einzugehen.

\section{Perioperative Measures for the Example of the Elderly Patient}

Perioperative nursing care of the elderly patient begins already with the decision to operate. Perioperative nursing care is understood as the networking and bringing together of nursing activities before, during and after an operation. Perioperative nursing care includes the preparation and supervision of patients waiting for an operation, all nursing activities during the surgical intervention through to the return of the patient to the ward. In this situation a structured nursing process is advantageous so that the steps and structures in the operating room and comprehensible and transparent for all involved personnel. An open exchange of information between the surgeon, patient, OR nurses and anaesthesiologists must be guaranteed. This is especially important for the elderly patient since the outcome of the operation may lead to substantial changes in daily life. All possible positive as well as negative consequences of the operation must be taken into consideration and discussed prior to surgery. In order to achieve the best possible treatment, it is essential in the preparation phase to create optimal working conditions not only for the surgical team but also for the anaesthesiology team and for their mutual cooperation. Experience shows that an imminent surgical intervention often induces feelings of insecurity and anxiety in the patient; this must be taken into consideration in the perioperative nursing process. Unfortunately, however, the short time available prior to the operation is usually not enough to provide the patient with a sufficiently empathic and caring atmosphere.

\section{Einschleusen}

Der erste Kontakt des OP-Pflegepersonals mit dem geriatrischen Patienten findet an der Patientenschleuse statt [3]. Vor diesem Erstkontakt sollte geklärt sein, ob bei dem Patienten eine Infektion oder Kolonisation mit resistenten Erregern (z.B. MRSA, VRE, ESBL oder 3MRGN) vorliegt, da in diesen Fällen spezielle hygienische Maßnahmen berück-

OP-JOURNAL 2014; 30: 46-49

(c) Georg Thieme Verlag KG Stuttgart · New York DOI http://dx.doi.org/10.1055/s-0034-1368242 sichtigt werden müssen, die u.a. eine Änderung des Einschleusungs- und Einleitungsprozesses zur Folge haben können (Tragen von Schutzkleidung, Narkoseeinleitung im Saal o.ä.). Bei geriatrischen Patienten, insbesondere aber bei Pflegeheimbewohnern sind Kolonisationen mit den genannten Erregern im Vergleich zur Allgemeinbevölkerung deutlich häufiger vorhanden, wenn auch nicht immer bekannt. Eine Erhöhung der Sicherheit kann bspw. durch geeignete Screening-Untersuchungen bei Aufnahme des Patienten erzielt werden. Bei bekannter Infektion oder Kolonisation ist dies in der Regel im Dokumentationssys- tem hinterlegt und ersichtlich und kann so bei Anmeldung des Patienten zur OP berücksichtigt werden. Im Zweifelsfall sollte eine telefonische Rücksprache mit der einweisenden Einheit (Station bzw. Ambulanz) erfolgen.

Das Vorgehen an der Patientenschleuse sollte wie folgt üblich sein [3]:

- Begrüßung und Vorstellung beim Patienten

- Identität des Patienten anhand der Akten vergleichen

- Vollständigkeit der Akten überprüfen

- Vergewisserung über die Nüchternheit des Patienten 
- mündliche Übergabe zwischen dem Pflegepersonal über pflegerische Probleme bzw. Ausnahmefaktoren (z.B. Dekubitus, Demenz etc.)

- Kontrolle, dass Hilfsmittel (z. B. Hörgeräte, Zahnersatz) und/oder Wertsachen (z. B. Ehering) abgelegt sind

Nach Überprüfung bzw. Durchführung dieser Schritte folgt das Einschleusen des Patienten.

Verschiedene, im geriatrischen Patientengut potenziell vorhandene Faktoren können dieses Vorgehen jedoch erschweren.

Insbesondere bei dementen oder verwirrten Patienten kann bereits die Identifikation des Patienten eine Herausforderung darstellen, da eine Orientierung zur Person und zur Situation häufig nicht gegeben ist. Weiter erschwert wird dies dadurch, dass verwirrte oder demente Patienten häufig keine Einsicht in die durchzuführenden Maßnahmen zeigen, wobei dies, insbesondere wenn der Patient unter Schmerzen leidet, nicht selten zu mangelnder Kooperation, Agitiertheit bis hin zu verbaler oder körperlicher Aggression vonseiten des Patienten führt. Auch zu Begleiterkrankungen, Allergien und Regelmedikation können ältere und demente Patienten häufig keine verlässlichen Informationen geben.

Die Vollständigkeit der Patientenakte, in der diese Informationen (z.B. nach fremdanamnestischer Erhebung) erfasst wurden, ist daher von entscheidender Bedeutung. Hilfreich für die eindeutige Identifikation des Patienten sind auch bei Aufnahme bzw. auf Station angelegte Armbänder mit entsprechendem Patientenetikett, aus dem Name und Geburtsdatum sowie ggf. Patientenidentifikationsnummer hervorgehen.

Aufgrund einer inaktivitätsbedingt verminderten Muskulatur („Sarkopenie“), der im Alter reduzierten Elastizität der Haut und einer verminderten lokalen Durchblutung weisen geriatrische $\mathrm{Pa}$ tienten häufig eine deutlich reduzierte mechanische Belastbarkeit der Haut auf. Dies bedingt, dass es bereits bei unsachgemäßer Manipulation (z.B. Ziehen am Patienten) rasch zu Riss- oder gar Décollement-Verletzungen kommen kann. Zudem ist das Risiko für Dekubitalulzera insbesondere bei präoperativ bereits immobilisierten oder eingeschränkt mobilen Patienten an aufliegenden Körperstellen erhöht.
Diesen Tatsachen muss bei jeglichen (Um-)Lagerungsmaßnahmen durch erhöhte Vorsicht und geeignete Polsterungsmaßnahmen Rechnung getragen werden. Da Dekubitalulzera bereits präoperativ vorliegen können, ist dies nicht zuletzt aus forensischen Aspekten bei der Übergabe zu erwähnen, der Hautstatus des Patienten nochmals zu überprüfen und präoperative Befunde schriftlich, wenn möglich auch mittels Fotodokumentation, festzuhalten.

Sollte der Patient Hilfsmittel oder Wertsachen mit in den OP bringen, ist dies schriftlich zu erfassen. Dabei ist Rücksicht geboten, denn der Patient fühlt sich möglicherweise sicherer mit Brille bzw. Hörgerät. Es sollte ihm daher ermöglicht werden, diese auch bis zur Narkose zu tragen, wenn dies nötig ist oder es dem Wunsch des Patienten entspricht. Die Prozedur der Ansprache ist demnach gegenüber einem jüngeren Patienten zu ändern. Fragt man laut und deutlich bei der Begrüßung, ob der Patient sein Gegenüber gut sieht und gut hört, findet man meist da schon heraus, inwieweit der Patient auf seine Hilfsmittel angewiesen ist.

Ein weiterer wichtiger Aspekt ist, ob der Patient im Rahmen früherer Eingriffe bereits Implantate erhalten hat (Osteosynthesematerial, Gelenkprothesen, oder anderes?, an welcher Lokalisation?), denn dies kann für die Lagerung oder aber das Anbringen einer Neutralelektrode bei Verwendung monopolarer Diathermiegeräte bedeutsam sein. Ähnliches gilt für Herzschrittmacher, wobei hier auch die Art des Schrittmachers bedeutsam ist. Hiervon hängt ab, ob überhaupt monopolare Diathermieverfahren angewendet werden dürfen. Hilfreich sind hierbei Schrittmacherpässe. Im Zweifelsfall sollte sicherheitshalber immer ein bipolares Diathermieverfahren angewendet werden.

Während sämtlicher perioperativer Maßnahmen ist auf die Bedürfnisse des Patienten fortwährend einzugehen, er sollte die ganze Zeit, vorzugsweise mit warmen Tüchern, bedeckt sein. Auch die Kommunikation mit dem Patienten muss bestehen.

Oft möchte der geriatrische Patient ein erhöhtes Kopfteil und Unterstützung mittels einer Knierolle, damit die Beine angewinkelt bleiben können. Dieses sollte beim Einschleusen als selbstverständlich angeboten werden. Der Patient ist oft verunsichert und gehemmt in der frem- den Umgebung. Der Schleusenprozess gestaltet sich sowohl in Bezug auf die Vorbereitung als auch auf die Durchführung aufgrund der genannten Faktoren bei geriatrischen Patienten in der Regel deutlich länger als bei jungen vitalen Patienten. Alle Handlungen werden individuell abgestimmt und haben dadurch längere Durchführungszeiten und besonderen Personalbedarf, z.B. bei Umlagerungen. Dies sollte bei der Planung der Prozesse berücksichtigt werden, um einen reibungslosen Ablauf zu gewährleisten.

\section{Lagerung}

Die Lagerung beginnt schon beim Einschleusen. Das Wichtigste ist, wie bereits erwähnt, dass der Patient durch Spezialauflagen (z.B. Tempurtische) gut gepolstert ist.

Wärmematten sollten zum Einsatz kommen, um eine Auskühlung des Patienten zu vermeiden, da eine Unterkühlung des Patienten u.a. eine Minderdurchblutung der Haut zur Folge haben und somit das Dekubitusrisiko erhöhen kann [1].

Ebenso sollte eine intraoperative Lagerungsänderung durch das Verschieben des relaxierten Patienten vermieden werden.

Da in der Alterstraumatologie die proximalen Femurfrakturen eine der am häufigsten operativ $\mathrm{zu}$ versorgenden Frakturen darstellen, beschreiben wir im Folgenden beispielhaft die Extensionslagerung anhand des Standards der Klinik für Orthopädie und Unfallchirurgie des Universitätsklinikums Freiburg.

Vorbereitung des Extensionstischs [2]

- Der OP-Tisch ist gereinigt und vorbereitet.

- Der OP-Tisch ist auf Funktionstüchtigkeit durch examiniertes Personal überprüft.

- Der entsprechende OP-Tisch wird im OP-Tagesplan durch examiniertes Pflegepersonal dokumentiert.

- Auflegen des Polstermaterials zur Dekubitusprophylaxe

- Ein Röntgenschutz wird so aufgelegt, dass die Durchleuchtung möglich ist.

- Extensionstische werden mit jeweils 2 Tüchern bezogen. Auf den Extensionstisch wird im Beckenbereich zum Herunter- oder Hochziehen des Patienten ein Laken quer aufgelegt.

- Anbringen einer Armlagerungsvorrichtung auf der "gesunden Seite“ am OP-Tisch und Auflegen eines Polsterkissens der entsprechenden Größe 
- Ein Bein- und Armgurt, für die "gesunde Seite“, und ein Kopfkissen werden in Reichweite bereitgelegt.

- Kennzeichnung des vorbereiteten OPTisches mit entsprechender Karte für den jeweiligen OP-Saal und die jeweilige OP

- Besonderheiten bei der Vorbereitung der OP-Tische oder Abweichungen dieser Beschreibung werden in den jeweiligen Prozessbeschreibungen aufgeführt.

- Beide Extensionsholme werden zum Fußende herunter geschwenkt.

\section{Vorbereitung des OP-Tisch-Zubehörs}

Der OP-Tisch wird mit den vorgegebenen und weiteren bedarfsorientierten Hilfsmitteln vorbereitet. Ausschlaggebend ist der körperliche Zustand des Patienten, darauf werden alle Handlungen ausgerichtet.

\section{Lagerung des Patienten [2]}

- Grundsätzlich erfolgt die Lagerung unter Wahrung der körperlichen und psychischen Sicherheit und Unversehrtheit des Patienten.

- Wache Patienten werden über alle Lagerungsmaßnahmen informiert.

- Die Intimsphäre des Patienten wird stets gewahrt.

- Der Patient wird in physiologischer Körperhaltung gelagert und gleichzeitig ein optimaler Zugang für den Operateur zum OP-Gebiet ermöglicht.

- Die Lagerung erfolgt durch eingewiesenes Pflegepersonal und den Arzt.

- Der Arzt trägt die Verantwortung für die Lagerung.

- Die Lagerungsart ist im OP-Tagesplan dokumentiert.

- Notwendige Lagerungshilfsmittel werden geplant eingesetzt und sind bei Lagerungsbeginn bereitgestellt. Lagerungshilfsmittel für die jeweilige OP sind in den entsprechenden Prozessbeschreibungen aufgelistet.

- An der Lagerung beteiligte Personen stehen zeitgerecht bereit.

- Der Lagerungsablauf erfolgt systematisch und effizient.

- Vor, während und nach der OP wird der Patient stets ausreichend gewärmt; dazu stehen vorgewärmte Decken, Wärmematte und Warmluftgeräte zur Verfügung.

- Zur Dekubitusprophylaxe wird adäquates Polstermaterial eingesetzt (Abb. 1).

- Um eine Durchfeuchtung der OPTischauflage während der Reinigung und Desinfektion des OP-Gebiets zu

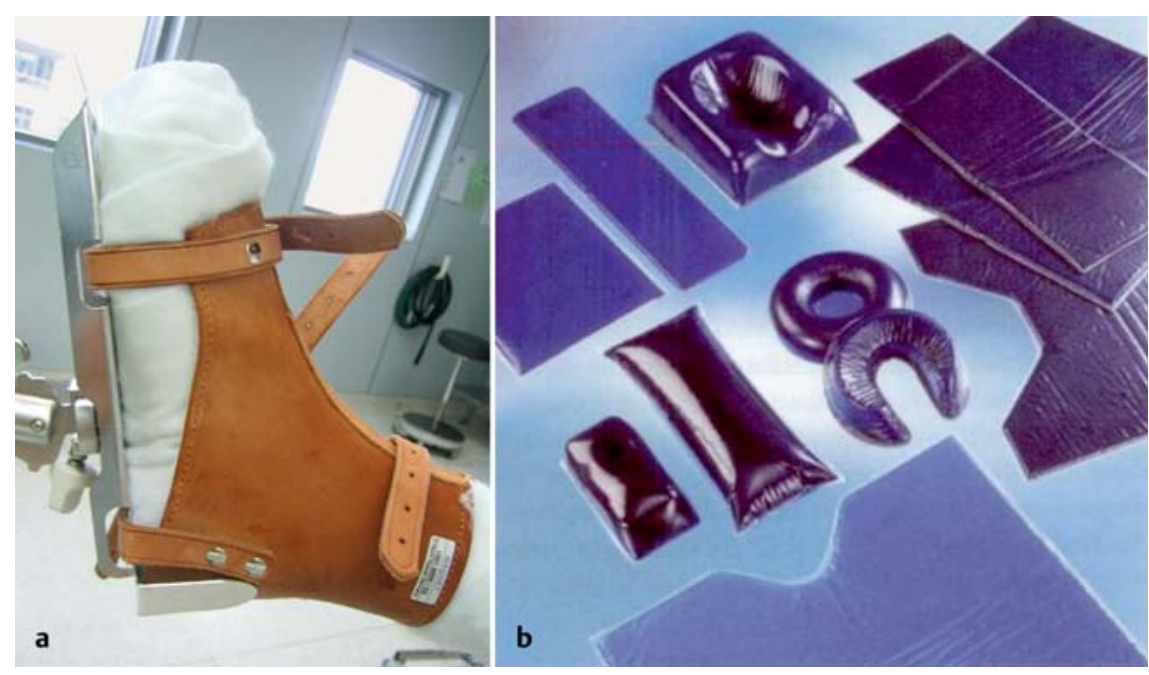

Abb. 1 Polstermaterial zur Dekubitusprophylaxe.

vermeiden, wird ein flüssigkeitsundurchlässiges Tuch kombiniert mit einem Stofftuch unter das OP-Gebiet geschoben.

- Die Rasur des OP-Feldes erfolgt nach den Rasurrichtlinien in der Regel im OP.

- Das OP-Feld wird bei allen Patienten mit einer antiseptischen Seife und Alkohol vorgereinigt. Zehenzwischenräume und liegende Fixateurs externes werden bei der Vorbereitung des Patienten mit Desinfektionsmittel eingesprüht.

- Bei der Applikation der Neutralelektrode sind die Bestimmungen und Sicherheitshinweise des Gerätebuchs des HF-Geräts zu beachten.

- Die Neutralelektrode wird mit ihrer ganzen Fläche, dem OP-Gebiet so nah wie möglich und zuverlässig an geeigneter Stelle auf der Haut des Patienten angebracht. Dabei ist zu beachten, ob der Patient schon liegende Implantate hat. Der Hautstatus des Patienten ist zu berücksichtigen, oft ist der geriatrische Patient kachektisch und exsikkiert, eine geeignete Stelle ist mit Bedacht zu wählen.

- Geeignete Stellen zur Applikation sind Körperregionen mit ausreichender muskulärer Deckung. Ungeeignete Gewebestrukturen sind Fett und Knochen.

- In Bereichen von liegenden Endoprothesen oder Implantaten werden keine Neutralelektroden angebracht.

- Zur Vermeidung unbeabsichtigter thermischer Gewebeschädigung wird der Kontakt des Patienten zu elektrisch leitfähigem Material streng vermieden.

- Am Körperstamm anliegende Extremitäten oder Haut-an Haut-Berührun- gen werden durch Zwischenlegen von trockenen Tüchern gegeneinander isoliert.

- Der „Infusionsarm“ wird in physiologischer Stellung ausgelagert, in Rückenlage nicht unter Körperniveau abgesenkt, maximal $90^{\circ}$ abduziert, im Ellenbogen leicht gebeugt und in Supination auf gepolsterter Armlagerungsschiene fixiert. Auf eine ausreichende Polsterung im Bereich des lateralen Epicondylus humeri bzw. Sulcus ulnaris ist zur Vermeidung einer Druckschädigung des N. ulnaris zu achten.

- Alle Lagerungsmaßnahmen erfolgen in Kooperation mit der Anästhesie.

- Detaillierte Lagerungsbeschreibungen sind in den entsprechenden Prozessbeschreibungen dokumentiert.

Die Extensionslagerung des Patienten erfolgt nach üblichem Standard. Ein Blasenkatheter ist vor Beginn der Lagerungsmaßnahmen anzulegen.

\section{Operationssaal: Saalarbeiten - Geräte}

Vorbereitung der Sterilcontainer und Einmalartikel nach Standard, hier Eventualitäten mit dem Operateur vorher klären und bereitstellen.

Anordnung der Instrumententische im OP-Saal

Anordnung der benötigten Geräte: HFGerät, Sauger, Röntgen C-Bogen.

\section{Installation des C-Bogens im OP-Saal}

Die Patientendaten am Monitorwagen eingeben. Das Gerät auf der „gesunden Seite“ positionieren, d.h. bei einer rechtsseitigen pertrochantären Femur- 


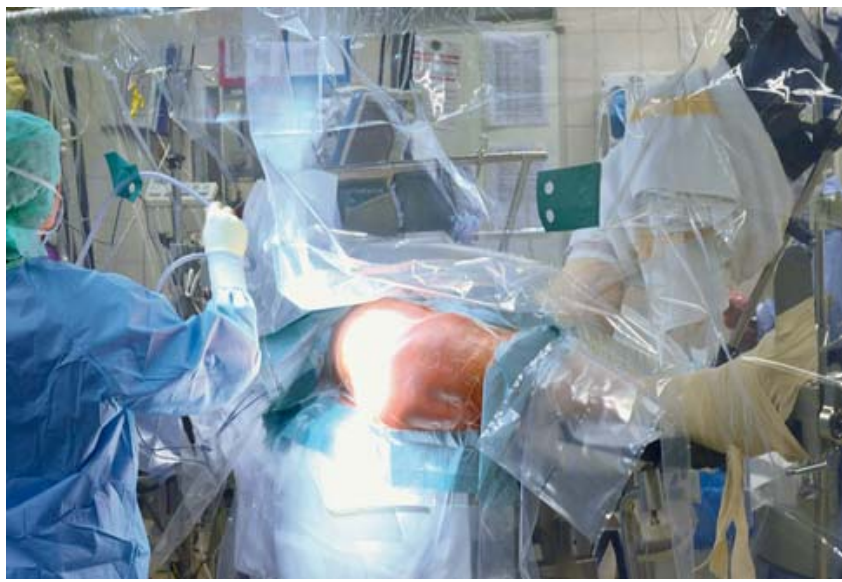

Abb. 2 Durchsichtiges Vertikaltuchset.

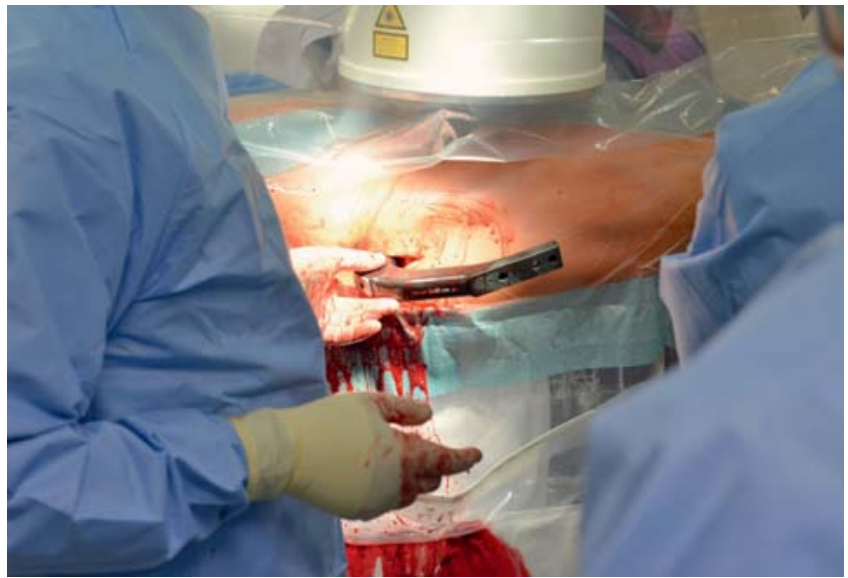

Abb. 3 Operation nach hausüblichem Standard. fraktur steht der C-Bogen auf der linken Seite. Die Bedienung des C-Bogens während der OP geschieht nur auf ärztliche Anordnung durch im Strahlenschutz unterwiesenes Personal (nach erfolgreich absolviertem Strahlenschutzkurs). Die Strahlenschutzmaßnahmen müssen dabei beachtet werden. Den Monitor am Fußende des Patienten platzieren, damit für das OP-Team während der Operation eine freie Sicht gewährleistet ist.

\section{Sterilabdeckung}

Die Abdeckung erfolgt mit einem durchsichtigen Vertikaltuchset (Abb.2), welches in vielen Häusern zum Standard gehört. Es erfolgt die Operation nach hausüblichem Standard unter Verwendung eines geeigneten Implantats (Abb. $\mathbf{3}$ ).

\section{Entlagerung}

Auch hier ist wieder ein besonderes Augenmerk auf den Hautstatus des Patienten zu legen. Die Klebeflächen der Abdeckung sollten sehr behutsam abgezogen werden, ebenso muss das Entfernen der Neutralelektrode vorsichtig geschehen, um Hautläsionen zu vermeiden.

Nach der Entlagerung ist der Hautzustand des Patienten zu kontrollieren. Veränderungen sind immer zu dokumentieren und Inhalt der Übergabe an die weiterbehandelnde Einheit (z.B. Aufwachraum, Intensivstation), damit frühzeitige Sekundärpräventionsmaßnahmen eingeleitet werden können, von den gesetzlichen Vorgaben abgesehen. Fersen und Sakralregion sind besonders gefährdet und sollten trotz vorausschauender Lagerung abschließend unbedingt überprüft werden. Auch ist der Entlagerungs- und Umlagerungsprozess postoperativ bei alten Menschen verlängert einzuplanen und im Bedarf mit höherer Personalkapazität durchzuführen.

\section{Zusammenfassung}

Zur Optimierung der Behandlungsabläufe bei angesichts der demografischen Entwicklung zu erwartenden zunehmenden Häufigkeit von Operationen bei geriatrischen Patienten muss diesen Patienten im OP-Ablauf gesondert Rechnung getragen werden. Räumliche und personelle Gegebenheiten müssen individuell angepasst sein, und es bedarf des Engagements aller Beteiligten im Behandlungsverlauf.

Beim geriatrischen Patienten müssen neben der eigentlichen operativen Versorgung einer Fraktur Komorbiditäten und altersbedingte Involutionsprozesse berücksichtigt werden, um ein optimales Behandlungsergebnis zu erzielen. Dies erfordert ein koordiniertes und auf die spezifischen Probleme des alten Patienten abgestimmtes Zusammenarbeiten aller beteiligten Berufsgruppen.

\section{Literatur}

${ }^{1}$ Kaltwasser B, Hierholzer G. Dekubitalulkusprophylaxe beim unfallverletzten älteren Menschen. OP-Journal 1995; 11: 89-96
${ }^{2}$ Dries R, Mutz A. Einführung von Qualitätsmanagement im traumatologischen OP. OPJournal 2005; 21: 82-89

3 Stauber E. Der geriatrische Patient im OP. München: Campus für Alten- und Krankenpflege; 1999

\section{Synke Petschinka}

OP-Schwester

Chirurgischer ZOP Bielefeld

An der Rosenhöhe 27

33647 Bielefeld

synke.petschinka@

klinikumbielefeld.de

\section{Regina Dries}

Gesamtleitung OP Pflege, im Zentralen OP-Bereich

Chirurgische Klinik

Universitätsklinikum Freiburg

Hugstetter Straße 55

79106 Freiburg i. B.

Priv.-Doz. Dr. med. Oliver Hauschild

Oberarzt, Facharzt für Orthopädie und Unfallchirurgie

Univ.-Prof. Dr. med.

Norbert Südkamp

Ärztlicher Direktor

Department Chirurgie

Klinik für Orthopädie und Unfall-

chirurgie

Universitätsklinikum Freiburg

Hugstetter Straße 55

79106 Freiburg i.B. 